\title{
Esterase Variants in Four Species of the Paramecium aurelia Complex ${ }^{1}$
}

\author{
SALLY LYMAN ALLEN, ELIZABETH T. LAU, THOMAS A. NERAD, ${ }^{2}$ and CAROLINE L. RUSHFORD \\ Division of Biological Sciences, The University of Michigan, Ann Arbor, Michigan 48109
}

\begin{abstract}
One hundred eighty-eight stocks of Paramecium primaurelia, $P$. biaurelia, $P$. tetraurelia, and $P$. octaurelia were grown axenically and tested for five esterases, visualized by starch gel electrophoresis, in a search for variant stocks. The five esterases can be distinguished on the bases of their substrate specificity, sensitivity to an inhibitor, and response to different growth conditions. This paper addresses the nature of the electrophoretic change in mobility of the variant stocks in order that species relationships can be more accurately assessed. Crosses carried out in all four species show that single genes determine the differences in mobility between variant and common subtypes. Extracts of variant stocks that gave similar patterns were run against each other, tested for their sensitivity to the inhibitor, and the pattern was compared to that found in extracts of stocks with variant and common subtypes in other species. The majority of the variants in $P$. primaurelia, $P$. tetraurelia, and $P$. octaurelia show an electrophoretic mobility characteristic of a common subtype, or a variant, in another species. The same proportion of variant subtypes as common subtypes have mobilities similar to esterase subtypes found in other species. Of the four species examined in this paper, $P$. tetraurelia and $P$. octaurelia appear to be most closely related on the basis of shared esterase subtypes. In $P$. biaurelia the mobilities of most of the variants are unique, as are the common esterase subtypes in this species. $P$. biaurelia stands out as having the greatest number of esterase subtypes, with very few of them homologous to subtypes found in other species. This observation supports the idea of greater diversification of stocks within $\boldsymbol{P}$. biaurelia than for the other three species.
\end{abstract}

$\mathrm{T}$ HE Paramecium aurelia complex includes 14 sibling species that are morphologically similar but between which no gene flow occurs (18). For some time now we have been concerned with the distribution of the esterases visualized by starch gel electrophoresis in this species complex, with a description of the types of esterases present, and with the degree of variation within and among the 14 species (2-5). With the exception of $P$. biaurelia, we found that the frequency of intraspecies variation appeared to be low.

There are four types of esterases (A, B, C, and D) that can be distinguished on the bases of substrate specificity and sensitivity to the inhibitor, eserine sulfate (4). Genetic factors as well as conditions of growth determine the electrophoretic mobility or activity of individual esterases in each species $(5,8)$. The behavior of individual esterases permits us to identify at least five different esterases: Esterase A (EstA), Esterase B (EstB), Cathodal Esterase C $\left(\right.$ Est $\left._{\mathrm{C}}\right)$, Anodal Esterase C $\left(\right.$ Est $\left._{\mathrm{A}}\right)$, and Esterase D (EstD).

The mobilities of EstA, EstB, and $\mathrm{EstC}_{\mathrm{C}}$ are usually identical in the majority of stocks within the same species (2-5). For each type of esterase the electrophoretic form(s) found in the majority of stocks will be referred to as the "common" subtype. The same subtype may be common in more than one species, since there are a limited number of subtypes found for each type of esterase in the species complex (5).

This paper focuses on four of the sibling species, $P$. primaurelia, $P$. biaurelia, $P$. tetraurelia, and $P$. octaurelia, with the objective of examining as many as possible of the wild stocks growing axenically in each of the species to search for variants. The choice of these four species was deliberate. They differ in their degree of inbreeding, tolerance to various environmental stresses, content of endosymbiotic bacteria, ability to mate across species lines, and geographical distribution (13, 14, 16, 17). In addition, the size of the collection of stocks is large for each of these species, and more information about them is available than for others, especially for $P$. tetraurelia.

We address the question of the nature of the electrophoretic change in mobility of the variant stocks. Is their electrophoretic

\footnotetext{
${ }^{1}$ Supported by a research grant, GM-15879, from the National Institute of General Medical Sciences, U.S. Public Health Service. We thank Almuth $\mathrm{H}$. Tschunko for drawing the diagrams. This is the first in a series of papers dedicated to the memory of Tracy M. Sonneborn.

2 Current address: American Type Culture Collection, 12301 Parklawn Drive, Rockville, Maryland 20852.
}

mobility similar to that of a common subtype found in anothel species? Or, is their electrophoretic mobility unique? Answers to these questions are important for assessing species relation. ships. Knowledge of the types of changes, and their frequency permits accurate calculation of genetic distances between species and construction of evolutionary trees (1). The frequency o intraspecies variation will be considered in a subsequent com munication (9).

\section{MATERIALS AND METHODS}

Stocks. Most of the stocks listed in Table I were obtainec from the Sonneborn Collection, then housed at Indiana University and now housed at the American Type Culture Collec. tion, Rockville, MD. Cultures of this collection growing on living bacteria were kindly provided by Myrtle V. Schneller. A few stocks $(229 \mathrm{vW}, 151 \mathrm{vW})$ were obtained from W. J. van Wag. tendonk. Others were collected from the Ann Arbor area $\left(1^{\text {st }} \mathbf{S}\right.$ : $22-1-14,19,21,28$; 22-2-12, 30; 22-3-2, 3, 4, 5, 6, 7, 11; 22-4-8; 33-3). Cal 1958 and NG-1 were stocks of paramecia unidentifiec as to species. Stocks growing on living bacteria were trans. ferred to axenic culture, some by means of the TREE and/or handwashing (4), most by a method described by Allen \& Nerad (7). Axenic stocks were maintained on Maintenance Medium: which contains the same components as Axenic Medium (pro. teose-peptone, trypticase, $\mathrm{MgSO}_{4}$, yeast nucleic acid, TEM-4T stigmasterol, and vitamins) with the addition of autoclaved bac. teria. Details of these culture media have been described else where $(7,8)$.

Growth of cultures for extracts. All of the stocks were growr on Axenic Medium to the stationary phase (7-12 days, depend. ing on the growth rate of the particular stock) in 3-liter flasks or 1-liter diptheria toxin bottles (8). Some of the stocks were also tested after growth in Maintenance Medium or Adaptatior Medium (Peter's salts solution, autoclaved bacteria, stigmas. terol, and vitamins; see 7, for details).

Identification of esterases. For enzyme extracts, all cultures were grown at $23^{\circ} \mathrm{C}$ and harvested by centrifugation; extracts of the cell concentrates were made by freeze-thawing. Electro. phoresis of whole-cell extracts was carried out in $12 \%$ starch gels using Electrostarch as described previously $(2-4,6)$. Twc substrates were used: $\alpha$-naphthyl propionate and $\alpha$-naphthy butyrate. Details of the histochemical procedures have beer described previously (4).

Inhibitor studies. Eserine sulfate at two concentrations (10-: and $10^{-4} \mathrm{M}$ ) was used to identify different types of esterases Gels were preincubated for $30 \mathrm{~min}$ in buffer containing the in 
TABLE I. List of stocks.

\begin{tabular}{|c|c|}
\hline Species & Stocks \\
\hline$P$. primaurelia & $\begin{array}{l}16,26,41,43,60,61,74,90,103,119,129 \\
143,144,147,153,156,168,171,175,177 \\
180,181,182,183,192,217,241,243,244 \\
257,258,285,313,334,335,336,337,513 \\
540,551,555,561, \mathrm{Cal} 1958, \mathrm{NG}-1,1^{\text {st }} \mathrm{S}\end{array}$ \\
\hline \multirow[t]{2}{*}{ P. biaurelia } & $\begin{array}{l}1,4,5,7,8,9,11,12,21,23,28,30,34,35 \\
36,50,53,71,72,86,88,91,93,100,104 \\
114,115,122,149,160,179,185,187,193 \\
197,206,207,208,234,235,249,259,260 \\
291,292,304,305,310,318,333,339,511 \\
517,526,527,537,560,562,563,564,570 \\
576,1010,1035,1038,1039,1041,1042 \\
1050, \text { Hu } 35-1, \text { B1 166-1 }\end{array}$ \\
\hline & $\begin{array}{l}22-1-14,19,21,28 ; 22-2-12,30 ; 22-3-2,3,4,5 \text {, } \\
\quad 6,7,11 ; 22-4-8 ; 33-3\end{array}$ \\
\hline P. tetraurelia & $\begin{array}{l}29,32,47,51,111,126,127,139,146,148 \\
151 \mathrm{vW}, 163,170,172,173,174,203,230 \\
239,242,277,278,280,298,315,316,322 \\
329, \mathrm{~A} 1, \mathrm{~A} 2\end{array}$ \\
\hline$P$. sexaurelia & 309 \\
\hline P. octaurelia & $\begin{array}{c}31,130,131,137,138,140,141,142,150,151, \\
202,213,214,216,218,224,229,252,276 \\
281,299 v W, 299 I, 300,307,327,330,565\end{array}$ \\
\hline$P$. tredecaurelia & 238 \\
\hline
\end{tabular}

hibitor. The solution was poured off and replaced with the incubation medium containing the inhibitor.

\section{RESULTS}

General aspects. In the Paramecium aurelia complex, five esterases can be distinguished on the bases of their substrate specificity, sensitivity to the inhibitor, eserine sulfate, and their response to different conditions of growth (type of medium, presence of acetate, presence of bacteria, etc.). Each stock was grown under conditions that would optimize the visualization of all five esterases in the gels. In some instances, different conditions of growth were used to enhance the appearance of a particular esterase. Extracts of each stock were run against extracts of a "standard" stock (having the common subtype for each esterase) for each of the species, and the mobilities of the esterases were compared. Variant stocks that gave similar patterns were run against each other, and the variant esterases were subsequently tested for eserine inhibition. To determine if the variant pattern was similar to that of a common subtype in another species, gels were run comparing the mobilities of the variant esterases with those of other species that were suspected of having similar mobilities. When it was clear that the variant pattern was unique, no further tests were necessary.

One hundred eighty-eight stocks were tested: 45 from $P$. primaurelia, 86 from $P$. biaurelia, 30 from $P$. tetraurelia, and 27 from $P$. octaurelia. Subtype I represents the common, or nonvariant phenotype, of each type of esterase in each species. Variant subtypes for each esterase type are designated II, III, etc. for each species. Table II summarizes the information on the esterase phenotypes of the stocks in the four species examined, and it gives the electrophoretic similarity of a particular phenotype to other species.

Esterase $A$. The A esterases react specifically with the substrate $\alpha$-naphthyl propionate and are inhibited by $10^{-3}$ to $10^{-4}$ $M$ eserine sulfate. The variant $A$ esterases in stocks $299 \mathrm{I}$ and 300 in $P$. octaurelia were sensitive to $10^{-3} \mathrm{M}$ eserine sulfate. So, too, were each of the A subtypes found in the $P$. biaurelia stocks. Typically, when grown in Axenic Medium, two to three, or more, isozymes are observed migrating to the anode, with the slowest isozyme having the greatest activity, and the second (and third) isozyme(s) having decreasing activity, in order of

TABLE II. Esterase phenotypes of stocks in four species.

\begin{tabular}{|c|c|c|c|c|c|c|c|c|c|c|c|c|}
\hline \multirow[b]{2}{*}{ Species } & \multicolumn{3}{|c|}{ Esterase A } & \multicolumn{3}{|c|}{ Esterase B } & \multicolumn{3}{|c|}{ Esterase $C_{c}$} & \multicolumn{3}{|c|}{ Esterase $C_{A}$} \\
\hline & $\begin{array}{l}\text { Sub- } \\
\text { type }\end{array}$ & $\begin{array}{l}\text { Electro- } \\
\text { phoretic } \\
\text { similarity }\end{array}$ & $\begin{array}{l}\text { Nos. of } \\
\text { stocks }\end{array}$ & $\begin{array}{l}\text { Sub- } \\
\text { type }\end{array}$ & $\begin{array}{c}\text { Electro- } \\
\text { phoretic } \\
\text { similarity }\end{array}$ & $\begin{array}{l}\text { Nos. of } \\
\text { stocks }\end{array}$ & $\begin{array}{l}\text { Sub- } \\
\text { type }\end{array}$ & $\begin{array}{c}\text { Electro- } \\
\text { phoretic } \\
\text { similarity }\end{array}$ & $\begin{array}{l}\text { Nos. of } \\
\text { stocks }\end{array}$ & $\begin{array}{l}\text { Sub- } \\
\text { type }\end{array}$ & $\begin{array}{c}\text { Electro- } \\
\text { phoretic } \\
\text { similarity }\end{array}$ & $\begin{array}{l}\text { Nos. of } \\
\text { stocks }\end{array}$ \\
\hline P. primaurelia & I & $\begin{array}{l}\text { sp. } 3,5,7,11 \text {, } \\
13,14 \\
\text { (I) }\end{array}$ & $45 / 45$ & $\begin{array}{l}\text { I } \\
\text { II } \\
\text { III }\end{array}$ & $\begin{array}{l}\text { sp. } 3,5(I) \\
\text { sp. } 6(I) \\
\text { sp. } 9(1)\end{array}$ & $\begin{array}{r}42 / 45 \\
2 / 45 \\
1 / 45\end{array}$ & $\begin{array}{l}\text { I } \\
\text { II } \\
\text { III }\end{array}$ & $\begin{array}{l}\text { sp. } 3,5,9,10,12(\mathrm{I}) \\
\text { sp. } 6(\mathrm{I}) \\
\text { sp. } 7,11,13(\mathrm{I})\end{array}$ & $\begin{array}{r}41 / 45 \\
2 / 45 \\
2 / 45\end{array}$ & $\begin{array}{l}\text { I (absent) } \\
\text { II }(7.5)^{\mathrm{b}}\end{array}$ & unique? & $\begin{array}{c}44 / 45 ? \\
1 / 45\end{array}$ \\
\hline P. biaurelia & $\begin{array}{l}\text { Ia } \\
\text { Ib } \\
\text { II } \\
\text { III } \\
\text { IV } \\
\text { V } \\
\text { VI } \\
\text { VIIa } \\
\text { VIIb } \\
\text { VIII } \\
\text { IX } \\
\text { X } \\
\text { XI } \\
\text { XII }\end{array}$ & $\begin{array}{l}\text { unique } \\
\text { unique } \\
\text { unique } \\
\text { unique } \\
\text { unique } \\
\text { unique } \\
\text { unique } \\
\text { unique } \\
\text { unique } \\
\text { unique } \\
\text { unique } \\
\text { unique } \\
\text { unique } \\
\text { sp. } 1 \text { (I) }\end{array}$ & $\begin{array}{r}32 / 86 \\
9 / 86 \\
12 / 86 \\
2 / 86 \\
1 / 86 \\
1 / 86 \\
1 / 86 \\
4 / 86 \\
7 / 86 \\
9 / 86 \\
1 / 86 \\
4 / 86 \\
1 / 86 \\
2 / 86\end{array}$ & $\begin{array}{l}\text { I } \\
\text { II }\end{array}$ & $\begin{array}{l}\text { unique } \\
\text { unique }\end{array}$ & $\begin{array}{r}79 / 86 \\
7 / 86\end{array}$ & $\begin{array}{l}\text { I } \\
\text { II } \\
\text { Absent? }\end{array}$ & $\begin{array}{l}\text { sp. } 8(\mathrm{I}) \\
\text { unique }\end{array}$ & $\begin{array}{r}75 / 86 \\
3 / 86 \\
8 / 86\end{array}$ & $\begin{array}{l}\text { I }(6.4) \\
\text { II }(6.2) \\
\text { III }(6.0) \\
\text { IV }(5.8) \\
\text { V }(5.6) \\
\text { VI }(5.4) \\
\text { VII (4.8) } \\
\text { VIII (absent) }\end{array}$ & $\begin{array}{l}\text { unique } \\
\text { unique } \\
\text { unique } \\
\text { unique } \\
\text { unique } \\
\text { unique } \\
\text { unique } \\
\text { - }\end{array}$ & $\begin{array}{r}21 / 86 \\
29 / 86 \\
24 / 86 \\
6 / 86 \\
1 / 86 \\
1 / 86 \\
1 / 86 \\
3 / 86\end{array}$ \\
\hline P. tetraurelia & I & sp. 8 (I) & $30 / 30$ & $\begin{array}{l}\text { I } \\
\text { II } \\
\text { III }\end{array}$ & $\begin{array}{l}\text { unique } \\
\text { sp. } 8 \text { (II) } \\
\text { sp. } 8 \text { (I) }\end{array}$ & $\begin{array}{r}27 / 30 \\
2 / 30 \\
1 / 30\end{array}$ & I & unique & $30 / 30$ & I (absent) & - & $30 / 30$ \\
\hline P. octaurelia & II & sp. 1 (I) & $25 / 27$ & II & sp. 4 (II) & $25 / 27$ & II & $\begin{array}{l}\text { sp. } 2 \text { (I) } \\
\text { unique }\end{array}$ & $\begin{array}{r}26 / 27 \\
1 / 27\end{array}$ & $\begin{array}{r}\text { I (6.8) } \\
(6.4) \\
\text { II }(6.6) \\
(6.2)\end{array}$ & $\begin{array}{l}\text { sp. } 2 \text { (I) } \\
\text { sp. } 2 \text { (II) }\end{array}$ & $\begin{array}{r}26 / 27 \\
1 / 27\end{array}$ \\
\hline
\end{tabular}

a sp. $3=P$. triaurelia, $4=P$. tetraurelia, etc. $I=$ common subtype found in majority of stocks.

b Distance in $\mathrm{cm}$ from origin. 


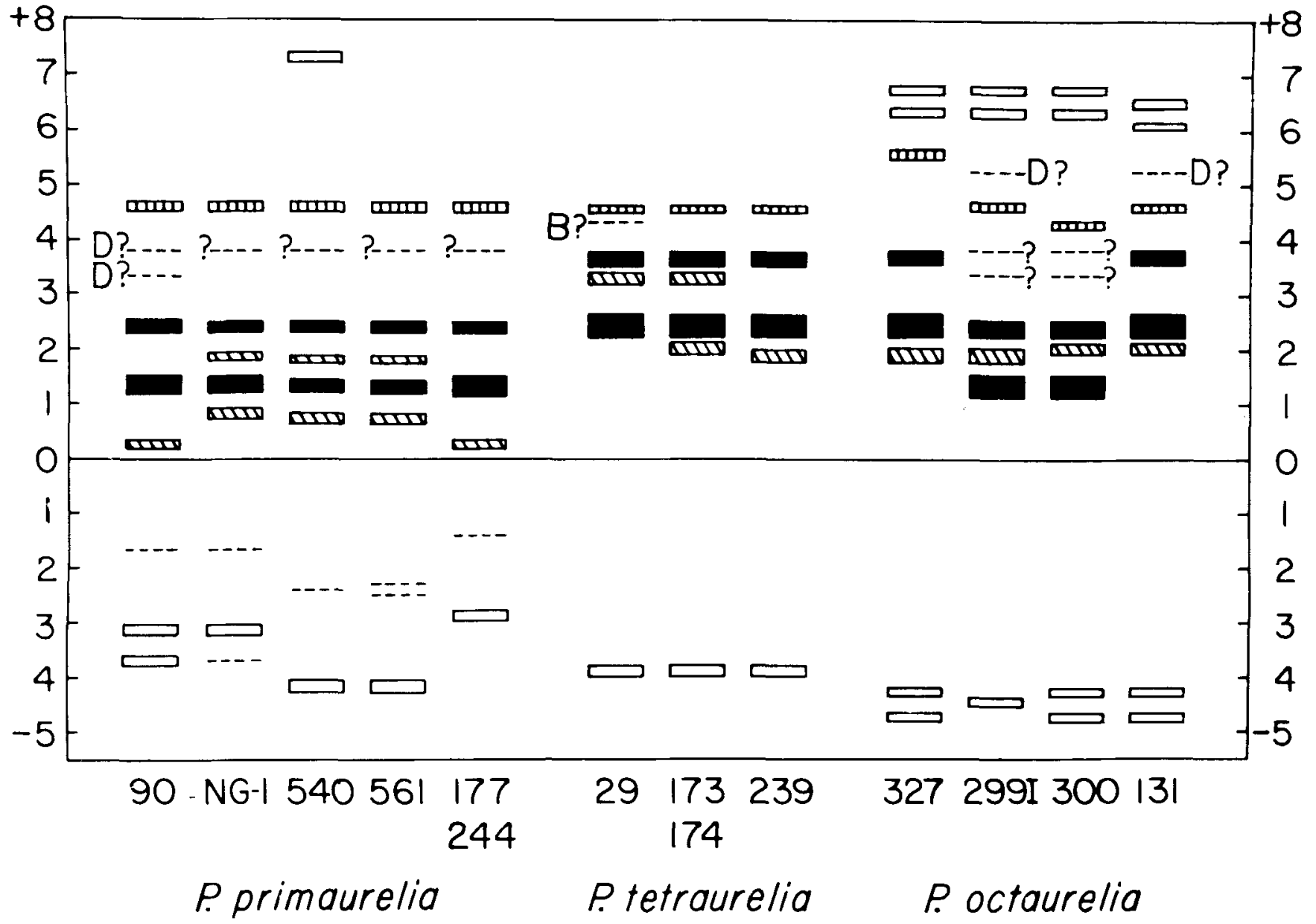

Fig. 1. Comparison of A, B, C, and D esterases in stocks with common and variant subtypes in $P$. primaurelia, $P$. tetraurelia, and ${ }^{0}$. octaurelia. Types of esterases are represented by the following symbols: $\square=\mathrm{A}$ type, $\mathrm{B}=\mathrm{B}$ type, $\square=\mathrm{C}$ type, and ail $=\mathrm{D}$ type. The dashed lines indicate esterases of very low activities. Distances in migration are marked off in centimeters from the origin (O) on the margins(s) of this and subsequent figures.

mobility. Growth in Adaptation Medium, or in living bacteria, may result in an enhancement of the activity of the more anodal isozymes.

No variant stocks were observed in $P$. primaurelia or $P$. tetraurelia for the A esterases. Two variant stocks; $299 \mathrm{I}$ and 300 , were identified in $P$. octaurelia. These stocks have A esterases that differ in mobility from the common subtype observed in the other 25 stocks (Fig. 1). The change in mobility appears to be identical in the two variant stocks, and the new phenotype is similar to subtype I found in $P$. primaurelia. Crosses between stocks $299 \mathrm{I}$ and 138 showed that this change is genetic (10); thus, the variant and commonly observed A esterases in $\boldsymbol{P}$. octaurelia are allozymes. Stocks $299 \mathrm{I}$ and 300 were both collected from Barro Colorado Island, Panama; thus, it is reasonable to conclude that both stocks carry the same mutation.

The number of A esterase isozymes in $P$. biaurelia is usually greater than in other species, commonly five to six, although some stocks (e.g., 563) may have only two, and others (e.g., 88 ) as many as seven isozymes. Some 12 , or more, different phenotypes can be distinguished among the 86 stocks tested. Figure 2 diagrams the $A$ esterase isozymes observed for each of the subtypes (I-XII) and gives the distribution of the stocks by subtype. Subtype Ib differs from Ia in having lower activity of the two most anodal isozymes. It is not clear that they are totally missing. Subtype VIIb has a similar relationship to VIIa. The most common subtype, Ia $+\mathrm{Ib}$, is found in 41 of the 86 stocks. It is also apparent that the A esterases are hypervariable in $P$. biaurelia since 45 of the stocks show 11 different phenotypes. Only subtype XII is similar in mobility to the A esterase isozymes commonly found in another species-specifcally those found in $P$. primaurelia, $P$. triaurelia, $P$. pentaurelia, $P$. septaurelia, $P$. undecaurelia, $P$. tredecaurelia, and $P$. quadecaurelia. All other subtypes appear to be unique. Crosses between stocks of different subtypes (e.g., 93 and 305) show that the difference in electrophoretic pattern is genetic (6), and that the subtypes are allelic products.

Esterase $B$. The B esterases can be detected by their greater preference for the substrate $\alpha$-naphthyl butyrate than $\alpha$-naphthyl propionate and by their greater resistance to the inhibitor eserine sulfate. The variant B esterases in stocks 540,561 , and NG-1 in $P$. primaurelia, 260,305, 318, and 560 in $P$. biaurelia, 173,174 , and 239 in $P$. tetraurelia, and 300 in $P$. octaurelia were resistant to $10^{-3} \mathrm{M}$ but sensitive to $10^{-2} \mathrm{M}$ eserine sulfate. Typically, when grown in Axenic Medium, only one electrophoretic form is observed, except for $P$. tetraurelia and $P$. 


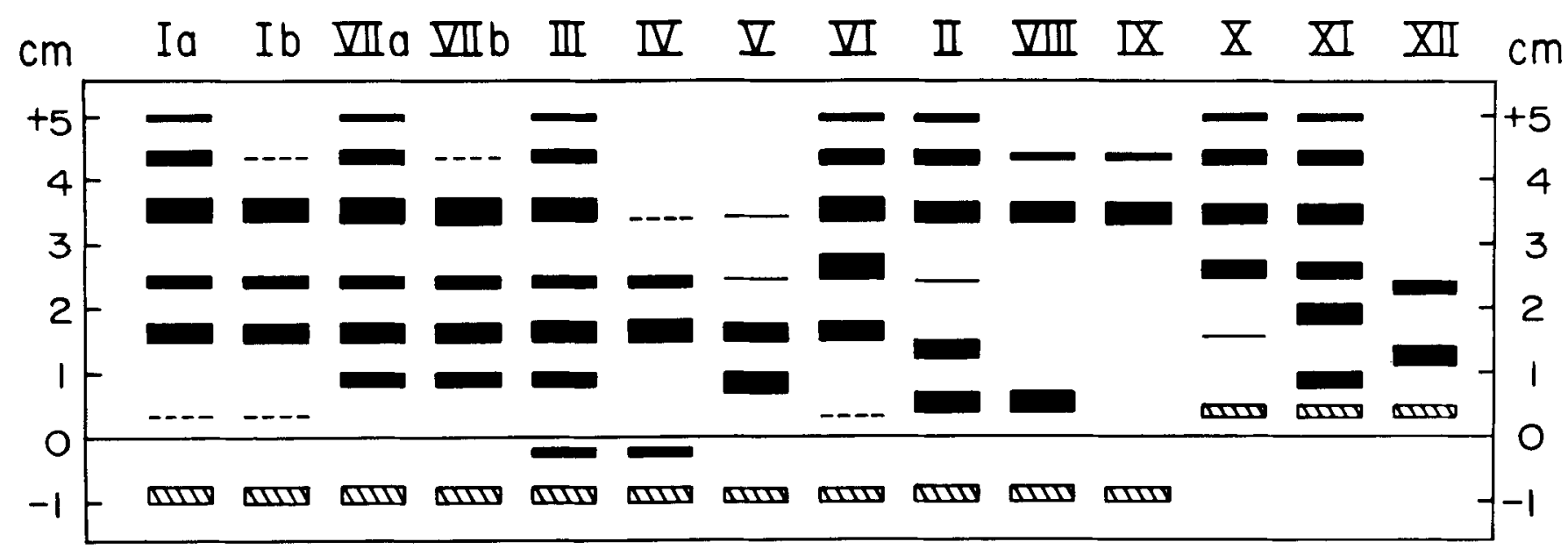

Fig. 2. Esterase A and B subtypes in stocks of $P$. biaurelia. The distribution of stocks by A subtype is, as follows: Ia-1, 5, 9, 11, $12,28,30$, $34,36,50,53,86,100,104,115,122,149,185,206,207,234,235,249,291,292,304,333,339,22-1-19,22-1-21,22-1-28,22-2-30$; $16-7,1010$, 1041 , 1042, BI-161-1, 22-2-12, 22-3-3, 22-3-6, 33-3; VIIa-23, 91, 179, 310; VIIb-511, 517, 537, 576, 1038, 1039, 1050; III-88, 22-1-14; IV-93; V-Hu $35-1$; VI-21; II-4, 8, 35, 71, 114, 160, 193, 197, 259, 562, 22-3-4, 22-3-5; VIII-72, 526, 527, 564, 570, 22-3-2, 22-3-7, 22-3-11, 22-4-8; IX-563; X-260, $305,318,1035 ;$ XI-208; XII-187, 560. Symbols $\square=$ A type, $=$ B type.

sexaurelia where two isozymes are often observed. Growth in Adaptation Medium with certain additions (proteose-peptone, yeast nucleic acid, and trypticase) enhances the activity of the second isozyme. Some of the variant stocks have two isozymes (540, 561, and NG-1 in $P$. primaurelia, and 173 and 174 in $P$. tetraurelia) when grown in Axenic Medium.

Variant stocks were observed in all four species. Three variant stocks, 540, 561, and NG-1, were identified in $P$. primaurelia. These stocks have $\mathrm{B}$ esterases that differ in mobility from the common subtype observed in the other 42 stocks. Figure 3a shows photographs of gels that compare the B esterases of stocks 90 and 540,561 and 540,540 and NG-1, and 540 and 309. Stock 90 has a B esterase typical of the common subtype in $P$. primaurelia. Stock 309 has B esterases typical of the common subtype in $P$. sexaurelia. The esterases in the $P$. primaurelia stocks are compared diagrammatically in Fig. 1. From such comparisons, we conclude that the change in mobility appears to be identical in 540 and 561 , but differs slightly from NG-1. The new phenotype in 540 and 561 is similar in mobility to subtype I of $P$. sexaurelia. NG-1 has a phenotype that is probably similar in mobility to subtype I of $\boldsymbol{P}$. novaurelia, although this comparison was not made. Crosses between stocks 90 and 540 showed that the change is genetic (10) and the products are allelic.

Seven variant stocks, $187,208,260,305,318,560$, and 1035 , were observed in $P$. biaurelia. These stocks have an anodally migrating $B$ esterase in contrast to the other 79 stocks that have a distinctive cathodally migrating $\mathbf{B}$ esterase (see Fig. 2). Photographs of gels showing some of the possible comparisons may be viewed in Fig. 3b: 206 and 1035, 208 and 305, 305 and 309, and 26 and 305 . Stock 206 has the cathodal B esterase typical of subtype I in $P$. biaurelia. B esterases typical of the common subtype for $P$. sexaurelia are found in stock 309 , for $P$. primaurelia in stock 26 . From such comparisons, we conclude that the change in mobility appears to be identical in all seven variant stocks, and that this new phenotype is unique. Crosses between stocks 50 or 93 and 305 showed that this change is determined by a single gene difference (6) and assorts independently of the A esterase differences. Thus, the variant and commonly observed B esterases in $P$. biaurelia are allozymes.

Three variant stocks, 173,174 , and 239 , were found in $P$. tetraurelia. These stocks have B esterases that differ in mobility from subtype I observed in the other 27 stocks. Figure $3 \mathrm{c}$ shows photographs of gels that compare the B esterases of stocks 173 and 29,174 and 173,173 and 239,327 and 174,327 and 239, and 173 and 300 . Stock 29 has B esterases typical of subtype I of $P$. tetraurelia. Stock 327 has a B esterase typical of subtype I of $P$. octaurelia, while stock 300 , also in $P$. octaurelia, is a variant. The esterases in these stocks are compared diagrammatically in Fig. 1. From such comparisons, we conclude that the change in mobility appears to be identical in 173 and 174, and this new phenotype is similar to a variant found in $P$. octaurelia. Stock 239 has a slightly slower B esterase, similar in mobility to the common subtype found in $P$. octaurelia.

Crosses were carried out between stocks 51 and 239. The mobilities of the $B$ esterases observed in the parents, $F_{1}$ exconjugants ( $F_{1}$ and $F_{1}^{\prime}$ are from the same mating pair), and exautogamous $\mathrm{F}_{2}$ 's are illustrated in Fig. 4. Segregation of the 51 and 239 phenotypes occurs in the $F_{2}$ exautogamous clones, as shown in Table III; however, there is a deficiency of the 239 phenotype, with $2: 1$ rather than $1: 1$ ratios being observed. The difference is clearly genetic, and two alleles at a single locus may be involved. The deficiency of the $E s t B^{239} / E s t B^{239}$ genotype might be accounted for by a lower viability relative to wild type, since the viabilities after autogamy were low for the cross.

Two variant stocks, 131 and 300 , were observed in $P$. octaurelia, with $\mathrm{B}$ esterases slightly slower in mobility than the common subtype present in the other 25 stocks. Photographs of gels that compare the B esterases of stocks 327 and 299I, 327 and 300, 299I and 300, and 300 and 131, are shown in Fig. 3d, and 300 and 173 in Fig. 3c. Stocks 327 and 299I have a B esterase typical of subtype I in $P$. octaurelia, while stock 173 in $P$. tetraurelia is a variant. The esterases in these stocks are compared diagrammatically in Fig. 1. From such comparisons, we conclude that the change in mobility appears to be identical in the two variant stocks, and that this new phenotype is similar to a variant found in $P$. tetraurelia.

Cathodal esterase $C$. The cathodally migrating $C$ esterases have a slight preference for $\alpha$-naphthyl propionate as substrate over $\alpha$-naphthyl butyrate. They are resistant to $10^{-2} \mathrm{M}$ eserine sulfate. Variant EstC $C_{C}$ 's in stocks 177, 540, and 561 in $P$. primaurelia, 560 in $P$. biaurelia, and 299 I in $P$. octaurelia are also 
(a)

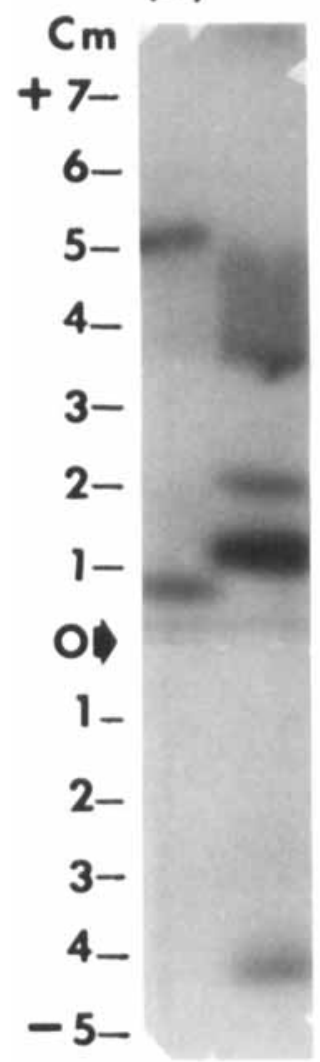

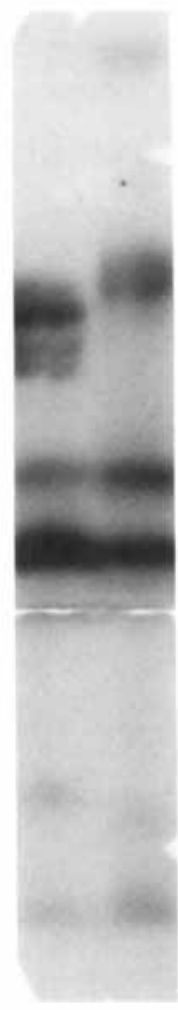

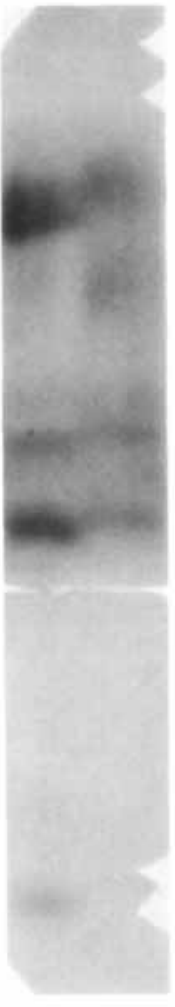

90

\section{$540^{540}{ }^{540}$ NG1 $6 / 309$

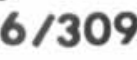

\section{P. Primaurelia}

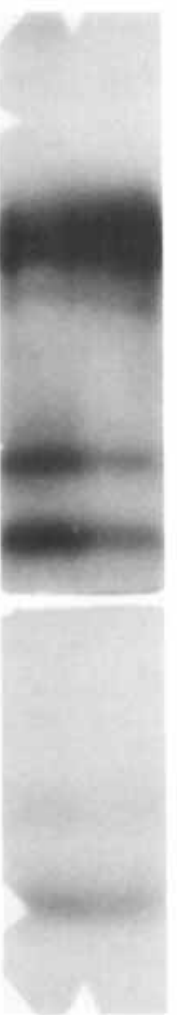

(b)
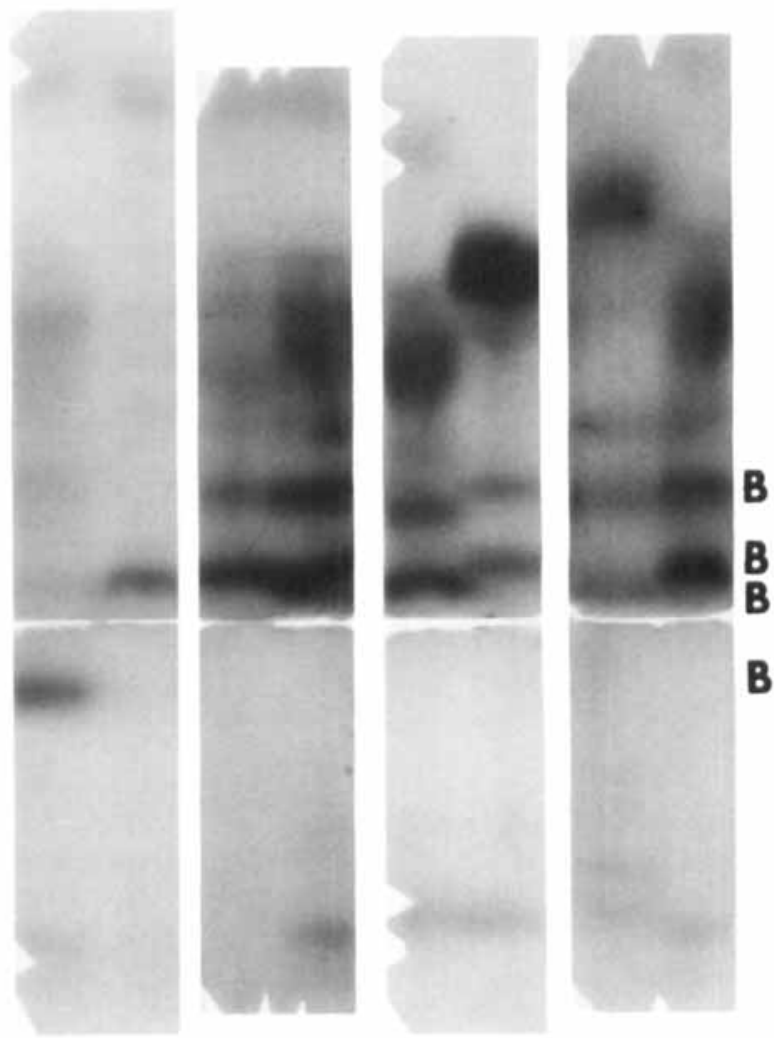

206 1035

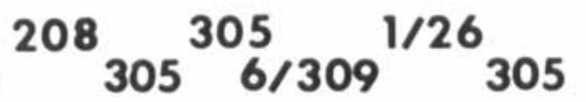

\section{P. biaurelia}

Fig. 3. Esterase B in the four species: (a) P. primaurelia; (b) P. biaurelia; (c) P. tetraurelia; (d) P. octaurelia. Where interspecies comparison is made, the number preceding the stock number refers to a particular species (e.g., $6 / 309$ refers to $P$. sexaurelia, stock 309 ). Substrate: $\alpha$-naphthyl butyrate.

unaffected by this concentration of eserine. The cathodal $\mathrm{C}$ esterases are particularly sensitive to low concentrations of acetate in the growth medium (8). Since the concentration of acetate (found chiefly in the component trypticase) in Axenic Medium decreases with the growth of the paramecia, older cultures show an increased activity of $\mathrm{EstC}_{\mathrm{C}}$. Even greater enhancement is observed when paramecia are grown in Adaptation Medium.

The common subtypes in six species of the $P$. aurelia complex need to be considered first, since further work has revealed differences in some of the relationships previously published (5). Two isozymes (a doublet) are found in $P$. primaurelia, $P$. biaurelia, and $P$. octaurelia; a single electrophoretic form is seen in $P$. tetraurelia, $P$. sexaurelia, and $P$. tredecaurelia. Comparisons of $8 / 327$ with $2 / 30,1 / 26$ with $2 / 206$, and $1 / 26$ with $8 / 327$ showed that the doublet is similar in mobility in $P$. biaurelia and $P$. octaurelia and faster than the doublet in $P$. primaurelia. The doublet in $P$. biaurelia and $P$. octaurelia is particularly sensitive to growth conditions, with the slower isozyme becoming much more active in older cultures. The singlet found in $P$. sexaurelia has about the same mobility as the slower of the two isozymes found in $P$. biaurelia and $P$. octaurelia, and migrates slightly faster than the singlet in $P$. tetraurelia, which has a mobility in between the slower of the two isozymes in $P$. biaurelia and $P$. octaurelia and the faster of the two isozymes in $P$. primaurelia. $P$. tredecaurelia has a singlet that migrates more slowly than the doublet in $P$. primaurelia.

No variant stocks were detected in $P$. tetraurelia. In $P$. primaurelia two variant stocks, 540 and 561 , were found to have a faster migrating singlet, and two other variant stocks, 177 and 244 , a slower migrating singlet than the doublet (subtype I) found in 41 other stocks (Fig. 1). The following stocks were compared: 90,26 , and 540; 540, 177, and 561; 540 with 2/206, 540 with $4 / 51,540$ with $6 / 309,540$ with $8 / 327$; and 177 with 13 / 238. From such comparisons, we conclude that the change in mobility appears to be identical in 540 and 561, and the new phenotype is similar to subtype I of $P$. sexaurelia. Crosses between stocks 90 and 540 showed that the change is genetic (10). In 177 and 244, a different change in mobility has occurred, which appears to be similar to subtype I of $P$. tredecaurelia.

Three variant stocks were detected in $P$. biaurelia: 187,560 , and 562 , which were similar in phenotype. These stocks have 


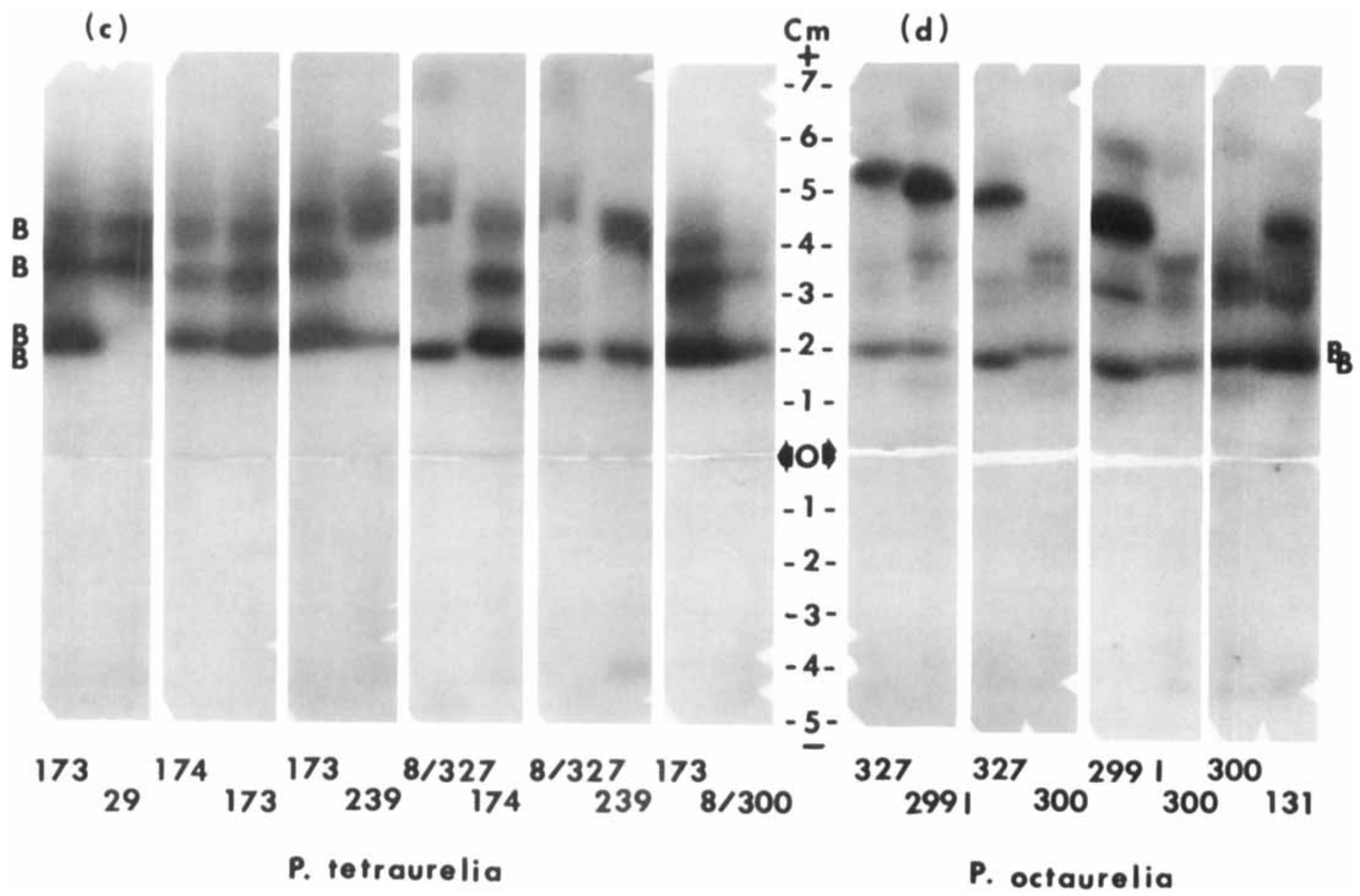

Fig. 3. (continued)

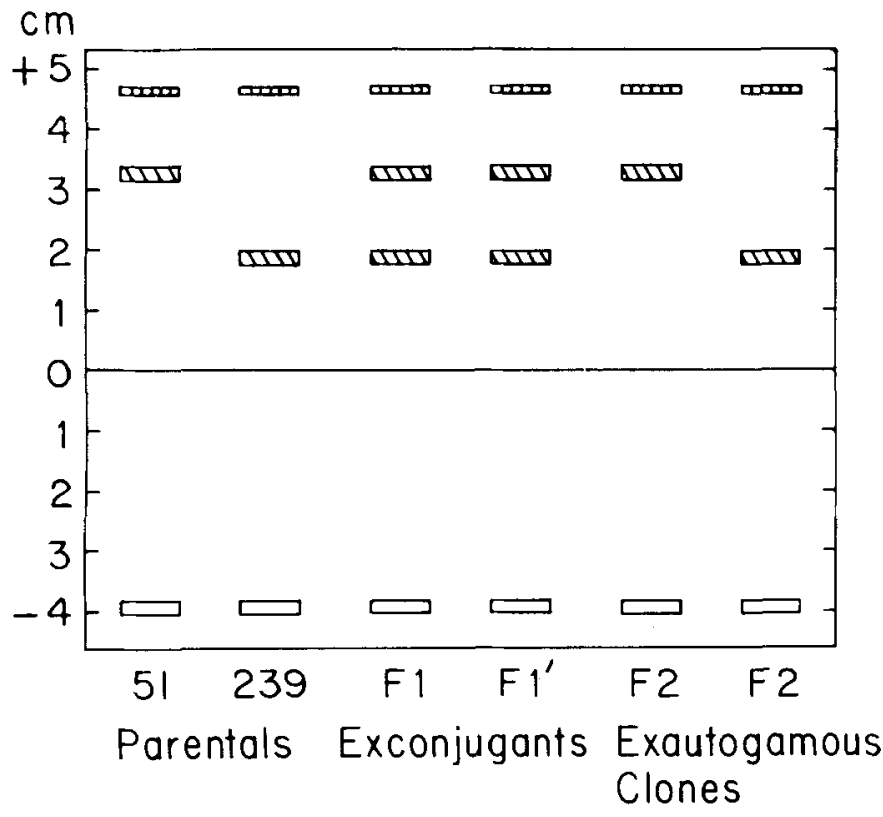

Fig. 4. The B esterases in $P$. tetraurelia, stocks 51 and 239 , and

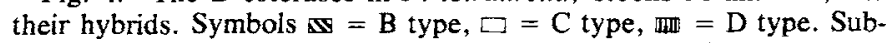
strate: $\alpha$-naphthyl butyrate. Five pairs of exconjugant clones were examined. Autogamy was induced in two of these sets, and two classes of $F_{2}$ exautogamous clones were observed among the progeny. a singlet, which migrates to a position that is similar in mobility to that of the fastest isozyme in $P$. primaurelia and slightly behind the slower isozyme in $P$. biaurelia.

One variant stock was observed in $P$. octaurelia: 2991 . This stock has a singlet migrating between the doublet seen in subtype I in this species (Fig. 1).

Anodal esterase $C$. The substrate preference, a greater affin-

TABLE III. Segregation of alleles $\mathrm{EstB}^{239}$ and $\mathrm{EstB}^{51}$ among $F_{2}$ exautogamous clones from crosses between stocks 239 and $51 .^{\mathrm{a}}$

\begin{tabular}{|c|c|c|c|c|}
\hline \multirow[b]{2}{*}{$F_{1}$ clone } & \multirow{2}{*}{$\begin{array}{c}\% \text { Viability } \\
\text { after } \\
\text { autogamy }\end{array}$} & \multicolumn{2}{|c|}{ Phenotype } & \multirow[b]{2}{*}{$\% 239$} \\
\hline & & 239 & 51 & \\
\hline $1 \mathrm{a}$ & 25 & 5 & 19 & 21 \\
\hline \multirow[t]{2}{*}{$1 b$} & 17 & 12 & 13 & 48 \\
\hline & & 17 & 32 & \\
\hline $2 a$ & 42 & 3 & 8 & 27 \\
\hline \multirow[t]{3}{*}{$2 \mathrm{~b}$} & 57 & 2 & 9 & 18 \\
\hline & & 5 & 17 & \\
\hline & Total & 22 & 49 & $31^{\mathrm{b}}$ \\
\hline
\end{tabular}

${ }^{a}$ Part of the data came from a Senior Honors Thesis, The genetic basis of Esterase B in Paramecium tetraurelia, presented by Richard J. Rygiel.

${ }^{b}$ Chi-square tests: $1: 1$ ratio, $P<0.01 ; 1: 2$ ratio, $P=0.5-0.7$. 
ity for $\alpha$-naphthyl propionate than for $\alpha$-naphthyl butyrate, and resistance to $10^{-2} \mathrm{M}$ eserine sulfate, identifies these esterases as $\mathrm{C}$ esterases. All are highly mobile and migrate to the anode, when present. In contrast to the cathodal $C$ esterases, the anodal $\mathrm{C}$ esterases are not affected by the level of concentration of acetate in the growth medium. In fact, they appear to be insensitive to acetate. They are, however, affected by other conditions of growth. They are most active in paramecia grown in Axenic Medium, and slightly active with growth in Maintenance Medium minus the component TEM-4T. They are much less active when paramecia are grown in Adaptation Medium.

Only stock 540 in $P$. primaurelia has this type of esterase. A singlet is observed, which migrates some $7.3-7.5 \mathrm{~cm}$ from the origin (see Fig. 1). Its activity appears to be unaffected by the growth cycle of the paramecia. In none of the stocks of $P$. tetraurelia has an anodally migrating esterase $\mathrm{C}$ been observed.

In contrast, all but three stocks of $P$. biaurelia have an anodal $C$ esterase. It usually migrates as a singlet $6.0 \mathrm{~cm}$ (24/86 stocks), $6.2 \mathrm{~cm}$ (29/86 stocks), or $6.4 \mathrm{~cm}(21 / 86$ stocks) from the origin, although slower migrating forms are found in nine other stocks. The distribution of the EstC $_{\mathrm{A}}$ phenotypes among the stocks appears to be uncorrelated with their EstA phenotype. The activity of these esterases is independent of the growth cycle of the paramecia.

All stocks of $P$. octaurelia have anodal $\mathrm{C}$ esterases in the form of a doublet. The slower isozyme increases with the age of the growth of the paramecia, whereas the faster isozyme appears to be independent of the growth cycle in its activity. In all stocks except 131, the two isozymes have mobilities of $6.8 \mathrm{~cm}$ and $6.4 \mathrm{~cm}$ from the origin. Both isozymes are slightly slower in mobility in stock 131 (see Fig. 1).

Esterase D. The D esterases have a slight preference for the substrate $\alpha$-naphthyl butyrate over $\alpha$-naphthyl propionate and are resistant to $10^{-2} \mathrm{M}$ eserine sulfate. They are very active in paramecia growing in Adaptation Medium and in Axenic Medium minus TEM-4T, but much less active in paramecia growing in complete Axenic Medium or in Maintenance Medium. Low activity in these latter media is correlated with the presence of TEM-4T (tartaric acid esters of beef tallow monoglycerides). They are inhibited by high concentrations of acetate in the medium and increase slightly with the age of the culture when paramecia are growing in Axenic Medium.

An esterase D that migrates anodally to about $4.7 \mathrm{~cm}$ from the origin is present in most stocks of $P$. primaurelia, $P$. biaurelia, $P$. tetraurelia, $P$. pentaurelia, $P$. sexaurelia, and $P$. octaurelia, and perhaps other species that have not been examined in detail. Other minor bands are often observed in the gels with the behavior of esterase $\mathrm{D}$. The $\mathrm{D}$ esterases are diagrammed in Fig. 1 for stocks in $P$. primaurelia, $P$. tetraurelia, and $P$.octaurelia. The $4.7 \mathrm{~cm}$ EstD is totally inhibited if living bacteria are added to the growth medium. This effect was observed in stock $29, P$. tetraurelia, as well as stock $540, P$. primaurelia, where it had been previously reported $(11,15)$.

It is not clear if there is stock variation in some of the species. However, some variation seems to occur in $P$. octaurelia, with stock 327 having a faster form of the major D esterase and stock 300 having a slightly slower one (see Fig. 1). There may also be some variation in $P$. tetraurelia, with stock 51 , for example, having a faster form of the major D esterase than stock 29.

\section{DISCUSSION}

Of the five different esterases, four are found in all four species of the $P$. aurelia complex examined here and in other species previously examined $(3,4)$. Only anodal esterase $C$ appears to have a spottier distribution among the species. Each type of esterase varies independently of the other types in different stocks within a species, although not all of the species examined here showed variants for a particular esterase. Single gene differences have been found for the mobility differences between stocks of the same species for the A esterases ( $P$. biaurelia: Ref. 5; $P$. octaurelia: Ref. 9) and for the B esterases ( $P$. primaurelia: Ref. $9 ; P$. biaurelia: Ref. 5; and probably $P$. tetraurelia: this paper). Variations among stocks in the thermal stability of the A or B esterases with similar mobility have been observed, implying further genetic differences (5). Differences in the activity of certain A or B esterases under different growth conditions have been noted $(6,10)$ and in the case of $P$. octaurelia they appear to be genetically determined. Both genetic as well as environmental factors control the mobility and expression of the cathodal $C$ esterases $(8,10)$.

What can be learned about the relationship of the electrophoretic forms of these esterases within and among species? Genetic data obtained from crosses within a species show that mutant and nonmutant forms of a single esterase are allelic products. Although similarity in mobility does not imply molecular identity, still, it is a criterion that can be used to compare mutant and nonmutant forms of an esterase across species lines. The esterase phenotypes of the stocks in the four species examined here are given in Table II, and any similarities in phenotype between species are noted. Such data are important in assessing species relationships so that more accurate evolutionary trees can be constructed (1).

With four esterases and four species there are a total of 16 possible comparisons of the most common, or nonmutant forms (labeled I in Table II), across the species complex. In $P$. primaurelia, $P$. tetraurelia, and $P$. octaurelia, $4 / 4,2 / 4$, and $2 / 4$ of the nonmutant forms of EstA, EstB, EstC $C_{C}$, and $E_{s t C}$ are similar to a nonmutant form in another species. This gives a total of $8 / 12$, or $67 \%$, showing similarity to another species. In contrast, $1 / 4$, or $25 \%$, of the nonmutants in $P$. biaurelia are similar to nonmutants in other species.

Are the mutant forms of the esterases in these four species more, or less, similar in mobility to subtypes found in other species? In $P$. primaurelia, $P$. tetraurelia, and $P$. octaurelia $4 / 5,2 / 2$, and $2 / 4$ of the mutant forms of Est $A, E s t B, E^{2} C_{C}$, or $\mathrm{EstC}_{\mathrm{A}}$ are similar in mobility to a mutant or nonmutant form in another species. Thus, $8 / 11$, or $73 \%$, of the mutants of these three species show a change characteristic of another species. $P$. biaurelia, with hypervariability for EstA and $\mathrm{EstC}_{\mathrm{A}}$, gives 19 non-I subtypes for the four esterases, and only $1 / 19$, or $5 \%$, of them are similar in mobility to another species. These data indicate that the same proportion of mutants as nonmutants is similar in mobility to esterase subtypes found in other species. This proportion is high for $P$. primaurelia, $P$. tetraurelia, and $P$. octaurelia, and most of the subtypes are not unique. The reverse is true for $P$. biaurelia, where most of the nonmutant and mutant subtypes are unique.

Tait (19-22) found variant stocks within different species of the $P$. aurelia complex with four of the seven enzymes he examined: isocitrate dehydrogenase (supernatant), isocitrate dehydrogenase (mitochondrial), $\beta$-hydroxybutyrate dehydrogenase (20), and glucosephosphate isomerase (22). Single gene differences were found for these variants $(19,21,22)$. If these enzymes are examined in the same way as for the esterases, but data from all 14 species are included, $47 / 56(84 \%)$ of the nonmutants and $7 / 17(41 \%)$ of the mutants are similar in phenotype to a mutant or nonmutant in another species. If data from only $P$. primaurelia, $P$. biaurelia, $P$. tetraurelia, and $P$. octaurelia are included, then $15 / 16(94 \%)$ of the nonmutants and $4 / 7(57 \%)$ of the mutants are similar to mutants or nonmutants in other species. Three of the mutants belong to $P$. biaurelia, and only $1 / 3$ is similar to another species in pheno- 
type. For these four enzymes, there is a marked difference in the proportion of mutants and nonmutants showing phenotypes similar to other species. Unlike the esterases, many more of the mutants show a unique phenotype.

Closer examination of mutant and nonmutant electrophoretic forms as to which species are involved reveals further relationships. Of interest are the qualitative similarities of mutant and nonmutant forms in $P$. tetraurelia and $P$. octaurelia. The nonmutant $\mathrm{A}$ esterases are similar in mobility. So also are the mutant $\mathrm{B}$ esterases despite the fact that the nonmutants are unique and differ between the two species. On the other hand, for the nonmutant EstC $\mathrm{C}_{\mathrm{C}}$ and mutant Est $\mathrm{A}$ in $P$. octaurelia, two other species are involved, namely $P$. biaurelia and $P$. primaurelia.

In contrast to the esterases, the "cross reactions" between or among species are much higher for the four enzymes studied by Tait (see above), if both nonmutants and mutants are considered. $P$. primaurelia shows similarities in electrophoretic mobility with all the other species except $P$. tetraurelia; $P$. octaurelia, with all species except $P$. tredecaurelia; and $P$. biaurelia, with all species except $P$. sexaurelia and $P$. tredecaurelia. A more limited set of cross reactions is observed for $\boldsymbol{P}$. tetraurelia, with similarities in mobility being observed for only nine of the 14 species.

Of the four species examined in this paper, $P$. tetraurelia and $P$. octaurelia appear to be more closely related on the basis of a number of features (16). Stocks in these two species are morphologically indistinguishable, and some stocks in both species harbor endosymbionts able to kill paramecia lacking them. Both species lack an immaturity period, have a short maturity period, and show group B mating type inheritance, all of which are adaptations that encourage inbreeding. Their serotypes cross react, they can mate with each other, and their spectrum of mating reactions with other species is similar. Moreover, they overlap in their geographical distribution, animals from both species even being found in the same body of water. They also share several esterase subtypes. Despite these similarities, no gene flow seems to occur since the $F_{1}$ hybrids, which are derived from only the $P$. octaurelia exconjugant, are all sterile (12).

$P$. primaurelia and $P$. biaurelia have longer immaturity periods and longer mature periods, adaptations that encourage more outbreeding, although they differ from each other in mating type inheritance (16). $P$. biaurelia has more kinds of symbionts than other species (14), and there appears to be greater diversity among the wild stocks in a number of characteristics than for any of the other known species of the $P$. aurelia complex (17). With respect to the esterases, there are more cross reactions of mutant and nonmutant subtypes in the species complex for $P$. primaurelia than for the other three species studied here. $P$. biaurelia stands out as having the greatest number of esterase subtypes, with very few of them homologous to subtypes found in other species. This observation supports the idea of greater diversification of the stocks within $P$. biaurelia than for the other three species. The next paper (9) in this series will examine the frequency of intraspecies variation in these four species.

\section{LITERATURE CITED}

1. Allen, S. L., Adams, J. \& Rushford, C. L. 1983. Interspecies relationships in the Paramecium aurelia complex: acid phosphatase variation. J. Protozool., 30. (in press)

2. Allen, S. L., Byrne, B. C. \& Cronkite, D. L. 1971. Intersyngenic variations in the esterases of bacterized Paramecium aurelia. Biochem. Genet., 5: 135-150.

3. Allen, S. L., Farrow, S. W. \& Golembiewski, P. A. 1973. Esterase variations between the 14 syngens of Paramecium aurelia under axenic growth. Genetics, 73: 561-573.

4. Allen, S. L. \& Gibson, I. 1971. Intersyngenic variations in the esterases of axenic stocks of Paramecium aurelia. Biochem. Genet., 5: $161-181$.

5. 1975. Syngenic variations for enzymes of Paramecium aurelia, in Markert, C. L., ed., Isozymes IV. Genetics and Evolution, Academic Press, New York, pp. 883-899.

6. Allen, S. L. \& Golembiewski, P. A. 1972. Inheritance of esterases A and B in syngen 2 of Paramecium aurelia. Genetics, 71: 469475 .

7. Allen, S. L. \& Nerad, T. A. 1978a. Method for the simultaneous establishment of many axenic cultures of Paramecium. J. Protozool., 25: $134-139$.

8. - 1978b. Effect of acetate on esterase $\mathrm{C}$ activity during the growth cycle of Paramecium. J. Protozool., 25: 273-279.

9. Allen, S. L., Nerad, T. A. \& Rushford, C. L. 1983. Intraspecies variability in the esterases and acid phosphatases for four species of the Paramecium aurelia complex. J. Protozool., 30. (in press)

10. Cavill, A. \& Gibson, I. 1972. Genetic determination of esterases of syngens 1 and 8 in Paramecium aurelia. Heredity, 28: 31-37.

11. Gibson, I. \& Cavill, A. 1973. Effects of bacterial products on a Paramecium esterase. Biochem. Genet., 8: 357-364.

12. Haggard, B. W. 1974. Interspecies crosses in Paramecium aurelia (syngen 4 by syngen 8). J. Protozool., 21: 152-159.

13. Nyberg, D. 1974. Breeding systems and resistance to environmental stress in ciliates. Evolution, 28: 367-380.

14. Preer, J. R., Jr., Preer, L. B. \& Jurand, A. 1974. Kappa and other endosymbionts in Paramecium aurelia. Bacteriol. Rev., 38: 113163.

15. Rowe, E., Gibson, I. \& Cavill, A. 1971. The effects of growth conditions on the esterases of Paramecium aurelia. Biochem. Genet., 5: $151-159$.

16. Sonneborn, T. M. 1957. Breeding systems, reproductive methods and species problems in protozoa, in Mayr, E., ed., The Species Problem, Am. Assoc. Adv. Sci. Symp., Washington, D.C., pp. 155324.

17. 1974. Paramecium aurelia, in King, R. C., ed., Handbook of Genetics, Plenum Press, New York, 2: 469-594.

18. 1975. The Paramecium aurelia complex of fourteen sibling species. Trans. Am. Microsc. Soc., 94: 155-178.

19. Tait, A. 1968. Genetic control of $\beta$-hydroxybutyrate dehydrogenase in Paramecium aurelia. Nature, 219: 941.

20. 1970a. Enzyme variations between syngens in Paramecium aurelia. Biochem. Genet., 4: 461-470.

21. 1970b. Genetics of NADP-dependent isocitrate dehydrogenase in Paramecium aurelia. Nature, 225: 181-182.

22. -1978 . Species identification in protozoa: glucosephosphate isomerase variation in the Paramecium aurelia group. Biochem. Genet., 16: 945-955.

Received 20 I 82; accepted 22 III 82 